\title{
Estudio descriptivo de los pacientes de riesgo y/o con úlceras por presión hospitalizados en una unidad de nefrología durante los años 2006 y 2007
}

Teresa Lope Andrea - María Isabel Durán Muñoz - María Rosario del Pino Jurado - Carmen Paterson Muñoz

Servicio de Nefrología. Hospital Clínico San Carlos. Madrid

\section{Resumen}

Por primera vez, en un Plan de Calidad de carácter nacional (Informe Eneas 2005), se hacen evidentes las úlceras por presión como un efecto adverso ligado a la hospitalización. Nuestro objetivo es conocer el perfil del paciente, su riesgo y el posterior desarrollo de úlceras por presión de los pacientes ingresados en la unidad de nefrología, definiendo el posible nivel de competencia, en cuidados de úlceras, del personal de enfermería.

Material y método: estudio descriptivo realizado en los años 2006-2007. Se obtuvieron los datos del programa informatizado de enfermería Gacela, se valoró a los pacientes mediante la escala de Norton, definiendo el plan de cuidados mediante metodología NIC y NOC.

Resultados: la incidencia del riesgo de ulceración al ingreso ha pasado de 26,3 a 49,7 en 2007. Desarrollan úlceras $7,4 \%$ de pacientes de riesgo en 2006 y $4 \%$ en 2007 . La edad media del paciente con úlcera se mantiene en hombres y aumenta en las mujeres. La mediana de días hasta conseguir curación de úlceras de Grado I empeora en 2007.

Correspondencia:

Teresa Lope Andrea

Servicio de Nefrología

Hospital Clínico San Carlos

C/ Profesor Martin Lagos s/n

28040 Madrid

elope.hcsc@salud.madrid.org
Conclusiones: las intervenciones de enfermería programadas son suficientes para prevenir el número de pacientes que no desarrollan úlceras y es preciso mejorar en curación de ulceras Grado I.

PALABRAS CLAVE:

- REGISTROS DE ENFERMERIA

- ÚLCERAS POR PRESIÓN

- PACIENTE NEFROLÓGICO

- PLAN DE CUIDADOS

Descriptive study of patients at risk and/or with pressure ulcers hospitalized in a nephrology unit in 2006 and 2007

\section{Abstract}

For the first time, in a Quality Plan with national scope (Eneas 2005 Report), pressure ulcers are shown to be an evident adverse effect associated to hospitalization. Our aim is to find out the patient profile, the associated risk and the subsequent development of pressure ulcers in patients hospitalized in the nephrology unity, defining the possible level of competence, in ulcer care, of nursing staff.

Material and method: descriptive study carried out in 2006-2007. The data were obtained from the Gacela nursing computer programme, patients were evaluated using the Norton scale, and the care plan was defined using NIC and NOC methodology. 
Results: the incidence of the risk of ulceration upon admission increased from 26.3 to 49.7 in 2007. In $2006,7.4 \%$ of patients at risk developed ulcers, and $4 \%$ in 2007 . The average age of the patient with an ulcer is stable in men and increases in women. The average number of days until Grade I ulcers are cured worsened in 2007.

Conclusions: programmed nursing interventions are sufficient to foresee the number of patients who do not develop ulcers and improvement is required in curing Grade I ulcers.

\section{KEY WORDS:}

NURSING RECORDS

PRESSURE ULCERS

NEPHROLOGY PATIENT

CARE PLAN

\section{Introducción}

Por primera vez, en un Plan de Calidad de carácter nacional (Informe Eneas 2005)릴 las úlceras por presión se hacen evidentes como un efecto adverso ligado a la hospitalización. Cuando nos referimos a úlceras por presión, incluimos lesiones de origen isquémico de profundidad variable, provocadas por apoyo directo de una prominencia ósea sobre la cama o sillón y maceraciones de piel por fricción o incontinencia.

El grado de práctica de enfermería en la prevención y tratamiento de las úlceras, se define en tres niveles:

- Nivel básico: personal con conocimientos teóricos deficientes, falta de destreza en el manejo del paciente de riesgo o con úlceras por presión, registros deficientes y/o no consulta con el experto. De este nivel se derivan acciones de mejora.

- Nivel de competencia: personal con conocimientos y experiencia en el manejo del paciente de riesgo y en la curación de úlceras por presión, con capacidad para demostrar juicio clínico en la práctica autónoma, registros adecuados y/o coordinación con el experto. De este nivel se derivan mejoras en la práctica.
- Nivel de excelencia: personal con nivel de competencia demostrado y que además se involucra en el avance del cuidado de enfermería a través de la formación, la educación y la investigación con autonomía o en coordinación con el experto. De este nivel se deriva el aumento del conocimiento a través de la investigación.

En los últimos años ha cambiado el perfil de los pacientes que ingresan en las unidades de hospitalización de nefrología; la mayoría son mayores de 65 años $^{3}$ y soportan períodos de inmovilización a causa de la comorbilidad asociada a su insuficiencia renal. El envejecimiento de nuestros pacientes junto a dicha comorbilidad y a su mayor supervivencia aumenta la dependencia, produciéndose una mayor necesidad de cuidados de enfermería. Por tanto, se realizan cuidados estandarizados o individualizados, encaminados a prevenir la aparición de úlceras o a conseguir buenos resultados en las mismas, en el caso de producirse a todos los pacientes de riesgo ingresados.

Los últimos datos recogidos del Grupo Nacional para el Estudio y Asesoramiento en Úlceras por Presión en España indican que un $5 \%$ del gasto sanitario anual de nuestro país se dedica a las úlceras por presión y un $26,6 \%$ del coste corresponde a los pacientes hospitalizados ${ }^{4}$. Este gasto viene definido por el coste de los materiales, el coste medio del tiempo de enfermería empleado en ese tratamiento durante el ingreso, que corresponde al $19,2 \%$ de su coste total, y la severidad de la lesión.

Nos planteamos, saber si la recogida de datos, ayuda a mejorar los cuidados en pacientes de riesgo y/o con úlceras por presión y a determinar el nivel de práctica enfermera. El objetivo de este trabajo es conocer el riesgo y el posterior desarrollo de úlceras por presión de los pacientes ingresados en la unidad de nefrología durante los años 2006 y 2007. Asimismo, se pretende inferir a la vista de los resultados, qué nivel de práctica alcanza el personal de la Unidad de Nefrología.

\section{Pacientes y Método}

Diseño y periodo de estudio: serie de casos retrospectiva realizada entre los años 2006 y 2007. 
Ámbito: el Hospital Clínico San Carlos es un hospital de tercer nivel encargado de atender a una población de referencia de 700.000 habitantes correspondiente al Área 7 de Madrid. El Servicio de Nefrología cuenta con 23 camas de hospitalización. El hospital dispone desde 1995 de una Unidad de Úlceras por Presión (UUPP) a cargo de dos enfermeras.

Sujetos de estudio: la población muestral son todos los pacientes ingresados en la Unidad de Nefrología en los años 2006 y 2007.

Plan de trabajo: Se recogieron los datos a partir del registro de enfermería informatizado Gacela. En dicho programa, se registran los datos de riesgo al ingreso y durante la estancia, en la gráfica de constantes (figura 1), y la aparición de nuevas úlceras y seguimiento de éstas, en el apartado de comentarios (figura 2) hasta el alta. La supervisora en un grafico manual, registra cada día a los pacientes nuevos que hay en cada nivel de la escala de úlceras y mensualmente lo trasmite a la UUPP que explota los datos de todo el hospital. Los informes elaborados con los datos recogidos por la UUPP se presentan anualmente a la supervisora y al personal de enfermería de la unidad.

La atención al paciente nefrológico se estructura de la siguiente forma: tras la valoración general del paciente y la especifica de las ulceras por presión aplicando la escala de Norton modificada por el hospital ${ }^{5}$ analizamos el contenido de los datos recogidos para elaborar un plan de cuidados, donde se contemplen los diagnósticos de enfermería relacionados con las úlceras por presión, el criterio de resultado y las intervenciones programadas para su resolución. Los posibles diagnósticos enfermeros ${ }^{6}$ son:

- Riesgo de deterioro de la integridad cutánea (riesgo de que la piel se vea negativamente afectada)

- Deterioro de la integridad cutánea (alteración de la epidermis, la dermis o ambas)

- Deterioro de la integridad tisular (lesión de la membrana mucosa o corneal, integumentaria o de los tejidos subcutáneos).

Las intervenciones enfermeras más comunes son: vigilancia de la piel, prevención de úlceras por presión (manejo de presiones, cuidados de la piel con tratamiento tópico y cuidados perineales), cuidados de la úlcera por presión, manejo de líquidos y manejo de la nutrición.

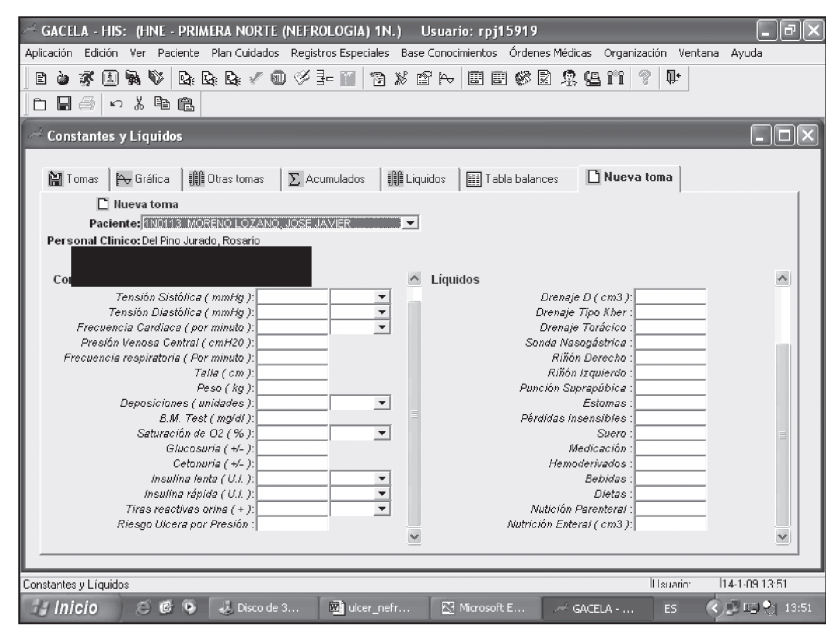

Figura 1. Pantalla del programa Gacela para el registro de constantes

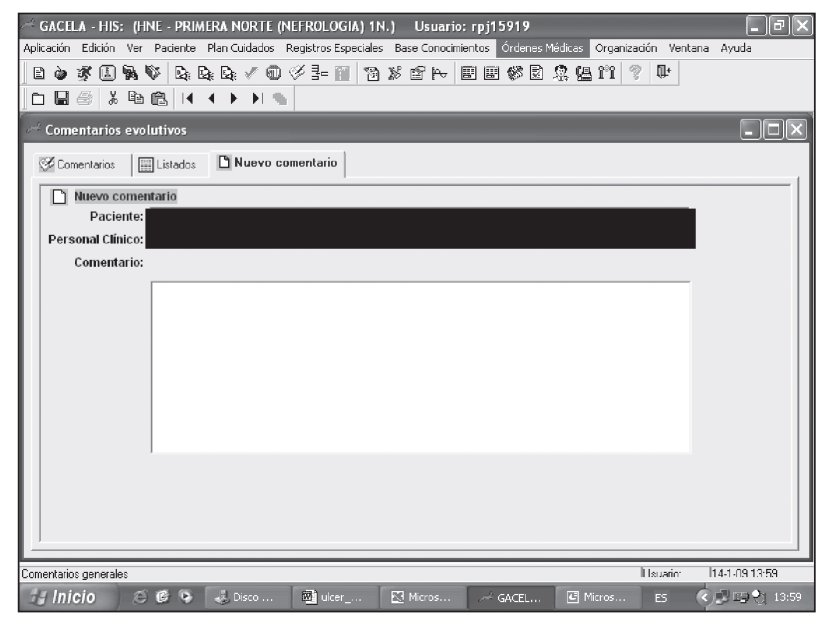

Figura 2. Pantalla para el registro de comentarios

La clasificación de las úlceras por presión se hace siguiendo los criterios de la Agency for Health Care Policy and Research ${ }^{7}$ : Grado I: eritema que no cede a la presión en 30 segundos y afecta a epidermis; Grado II: pérdida parcial del grosor de la piel afectando a epidermis y dermis superficial. Flictena exudativa; Grado III: pérdida del grosor de la piel implicando daño o necrosis del tejido subcutáneo que puede extenderse hasta la aponeurosis subyacente; Grado IV: pérdida del grosor completo de la piel con destrucción extensa; tejido necrótico o daño al músculo, hueso o estructuras de sostén.

\section{Variables:}

- Independientes: sexo, edad de los pacientes, incidencia de riesgos al ingreso a través de la medición del riesgo aplicando la escala de 
Norton modificada en este hospital en 1995 y número de casos que presentan riesgo de ulceración en la primera valoración al ingreso en la unidad ese año / número de ingresos ese año en la unidad.

- Dependientes: incidencia de ulceración como indicador de calidad a través del número de nuevas úlceras desarrolladas en la unidad ese año / número de pacientes en riesgo en la unidad y evento final (curación, estabilización o empeoramiento y número de días hasta curación).

Los observadores independientes de la Unidad de Úlceras por Presión mediante observación participante definieron el Nivel de Competencia del personal de la Unidad de Nefrología.

\section{Resultados}

En el año 2006 ingresaron en la unidad 722 pacientes, $61 \%$ hombres ( 440 ) y $39 \%$ mujeres (282); en el año 2007 ingresaron 660 pacientes, $69 \%$ hombres (457) y $31 \%$ mujeres (203). La estancia media en 2006 fue de 12,0 días y en 2007 de 12,7.

La incidencia de riesgo de ulceración al ingreso en 2006 fue $26,3 \%$ y en 2007 49,5\% ( $p<0,0001)$. Se desarrollaron úlceras en un $7,4 \%$ de los pacientes de riesgo en 2006 y en un $4 \%$ de los pacientes de riesgo en 2007 ( $p<0,0001)$. Presentaron úlceras 5 hombres y 6 mujeres en 2006 y 6 hombres y 5 mujeres en 2007. La edad media de los pacientes que presentaron úlceras en hombres se mantiene alrededor de los 70 años $(p=0,87)$, y en mujeres pasa de 63 años en 2006 a 76 años en 2007 ( $p<$ 0,001). (Figura 3).

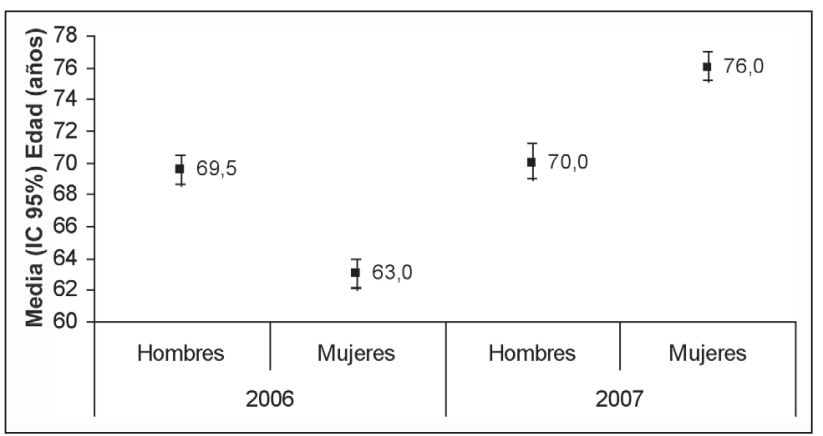

Figura 3. Distribución de edad de los pacientes que presentaron úlceras
En 2006, de 11 pacientes con úlceras presentaron riesgo moderado 7 y riesgo alto 4. En 2007, de 11 pacientes 7 presentaron riesgo alto y 4 riesgo moderado (figura 4). Los datos sobre la evolución de las úlceras producidas en la unidad, en 2007 se presentan en la tabla 1; no se dispone de estos datos en el 2006.

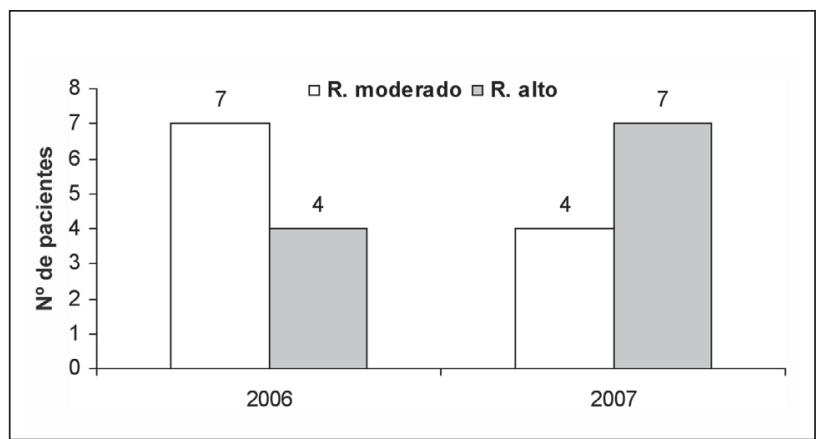

Figura 4. Distribución del riesgo en los pacientes con úlceras.

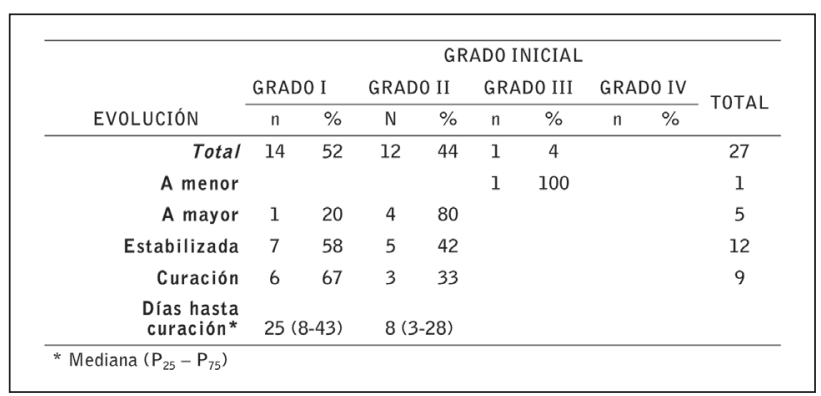

Tabla 1. Distribución de úlceras iniciadas y tratadas en la unidad en 2007.

La mediana de días necesarios hasta conseguir la curación de las úlceras de Grado II es menor en el año 2007 y en ambos años las úlceras de Grado II requieren un tiempo mediano inferior a las úlceras de Grado I (tabla 2).

\begin{tabular}{rcc}
\hline & Año 2006 & Año 2007 \\
& Mediana $\left(P_{25}-P_{75}\right)$ \\
Grado I & $22(5-26)$ & $25(8-43)$ \\
Grado II & $17(7-27)$ & $8(3-28)$ \\
\hline
\end{tabular}

Tabla 2. Días hasta la curación de la úlcera.

Basándose en la definición del nivel de práctica profesional, los observadores independientes de la Unidad de Úlceras por Presión mediante observación participante definieron que el personal de la unidad 
de nefrología alcanzaba un nivel 2 (nivel de competencia) en la práctica de enfermería en la prevención y tratamiento de las úlceras.

\section{Discusión y Conclusiones}

La distribución por sexo ha cambiado entre los años 2006 y 2007, el porcentaje de hombres aumentó y el de mujeres disminuyó. La incidencia del riesgo de ulceración al ingreso ha aumentado en un porcentaje clínicamente significativo, pero los cuidados preventivos de enfermería estandarizados o individualizados en pacientes de riesgo han sido eficaces dando lugar a una menor incidencia de ulceración.

La mediana de días hasta la curación en el Grado II es mayor en 2006, aunque la distribución por percentiles no parece significativamente distinta. En el año 2007 el número de días de curación de las úlceras de Grado I (eritema) es mayor, alcanzando un percentil 75 de 43 días, lo que podría indicar que se ha minimizado la importancia del inicio de la ulceración y/o realizado cuidados de enfermería deficientes.

El conocimiento y difusión mediante un informe anual de los datos relacionados con las úlceras por presión permite conocer el perfil de los pacientes nefrológicos que presentan úlceras, qué riesgo tienen todos los pacientes al ingreso, la tendencia del riesgo de cara al futuro (mujeres mayores de 70 años) y qué grado de ulceración desarrollan. Este conocimiento nos permite saber, por una parte, que las intervenciones enfermeras son actualmente suficientes para optimizar el número de pacientes que no desarrollan úlceras durante su ingreso, teniendo en cuenta el riesgo alto en pacientes con deficiente estado de la piel y/o la nutrición, neuropatías centrales o periféricas, déficit de movilidad, escasa o nula actividad o/e incontinencia fecal y plantear modificaciones en el abordaje de las úlceras de Grado I para optimizar los tiempos de curación.

Disponer de un registro de enfermería es irrescindible para garantizar la calidad de los cuidados $^{8}$, en nuestro caso, el registro unificado con la Unidad de Úlceras por Presión y la posterior difusión de los informes de resultado, permite la mejora de: la labor asistencial (continuidad de cuidados de cura), la gestión (gestión de recursos de alivio de presión, de tratamiento de la piel sana y de curación), la formación (si la incidencia de ulceración aumentara sin relación al riesgo) y la investigación (cómo se realiza el abordaje curativo de las úlceras Grado I y qué cambios se podrían abordar)

\section{Bibliografía}

1. Ministerio de Sanidad y Consumo. Estudio Nacional sobre efectos adversos ligados a la hospitalización. Informe Eneas. Febrero 2006. www.msc.es

2. SEEUE. Estándares para la práctica de enfermería de urgencias y emergencias. [Pagina principal en Internet]. Madrid: SEEUE; 2004[acceso 3de octubre de 2008]. Disponible en:

www.enfermeriadeurgencias.com/documentos/ fundamentos/estandares.pdf -

3. Arrieta J, Castro P, Gutiérrez Ávila G, Moreno Alia I, Sierra T, Estébanez C. Informe de situación de diálisis y trasplante en España 2004. Nefrologia 2007; 27(3): 279-299.

4. Soldevilla Agreda JJ, Torra i Bou JE, Verdu Soriano J. Epidemiología, coste y repercusiones legales de las úlceras por presión en España, años 20052006. Barcelona 2007. Smith \& Nephew.

5. Quiralte C, Martinez RM, Fernández C. Estudio de la validez de criterio de la Escala de Norton modificada del Hospital Clínico San Carlos. Enf Clin, 1998.8 (4):151-155.

6. Johnson M, Bulechek G, Butcher H, Maas M, McCloskey J, Dochterman J, Moorhead S, Swanson E. Interrelaciones NANDA, NIC y NOC. Diagnósticos Enfermeros, Resultados e Intervenciones. $2^{\mathrm{a}}$ ed. Elsevier. 2006.

7. Soldevilla Agreda JJ, Torra i Bou J.E, Atención integral de las heridas crónicas. Madrid: SPA; 2004.

8. Alfaro R. El pensamiento crítico en enfermería. Un enfoque práctico. Barcelona: Masson; 1997. 